\title{
Conceptos y códigos clave para médicos sin formación bioética: revisión bibliográfica
}

\author{
Key concepts and codes for physicians without \\ bioethical training: bibliographic review
}

Yeyetsy Ordóñez Azuara,* Raúl F Gutiérrez Herrera, ${ }^{\ddagger}$ Celina Gómez Gómez,
Melanie Susset Cisneros González," Diana Rocío González Vázquez"

\section{RESUMEN}

La bioética consiste en el estudio sistemático y científico de la conducta humana en el ámbito de las ciencias de la vida y la salud, las cuales se analizan a la luz de los valores y de los principios morales. Su conocimiento mejora la buena práctica médica. El objetivo del presente trabajo es identificar los códigos bioéticos relacionados con la medicina desde el punto de vista de la ética y la legalidad para el ejercicio cabal de la profesión médica actual en México. Material y métodos: Con el propósito de compilar de forma clara y estructurada la información respecto al tema, se realizó una búsqueda bibliográfica a través de diferentes fuentes de información, buscadores y bases de datos, entre ellas: Google Scholar, Medline, PubMed y SciELO. En el presente trabajo, se incluyeron las revisiones del tema, los artículos de investigación y los capítulos de libros; posteriormente, se hizo un análisis de contenido y selección de literatura por un grupo de expertos en bioética, el cual permitió elaborar un documento guía para el profesional de la salud, el cual nos permitirá conocer y atender de forma oportuna los aspectos relacionados con la bioética en la atención médica. Conclusiones: Los conceptos y códigos clave relacionados con el ejercicio cabal de la medicina en la asistencia médica desde la perspectiva bioética son: Declaración de Ginebra, Código Internacional de Ética Médica, Código de Conducta de la Secretaría de Salud, Código de Bioética para el Personal de Salud, Ley General de la Salud, las Normas Oficiales Mexicanas y las Recomendaciones generales para mejorar la práctica de la medicina de la CONAMED.

Palabras clave: Bioética, Código de Ética, revisión ética.

\begin{abstract}
The systematic and scientific study of human behavior in the light of life sciences and health, analyzed through values and ethical principles is what we call Bioethics. Knowing and doing bioethics improves the medical practice. The goal of the present work is to identify the bioethical codes related to medicine, from ethics and legality, for the full exercise of the medical profession today in México. Materials and methods: With the purpose of gathering in a clear and organized way the information regarding this subject, a biographical search was made through different information sources, search engines, and databases like, Google Scholar, Medline, PubMed, and SciELO. Subject reviews, research articles, and book chapters were included; afterward a content analysis and literature selection were made by bioethics experts, flowed by the creation of a guide document meant for health professionals. This guide will allow to know and attend in a timely manner the aspects related to bioethics in the medical care. Conclusion: The key concepts and codes related to the full exercise of medicine in medical care from a bioethical perspective are: Declaration of Geneva, International Code of Medical Ethics, Code of Conduct of Health Secretary, Bioethics Code for Health Professionals, General Law of Health, Official Mexican Norms and the General Recommendations to Improve Medical Practice by CONAMED.
\end{abstract}

Keywords: Bioethics, Codes of Ethics, ethical review.

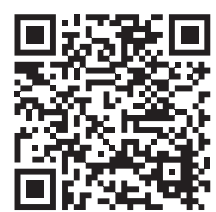

* Doctoranda en Bioética. Profesora del Departamento de Medicina Familiar del Hospital «José Eleuterio González». Universidad Autónoma de Nuevo León; Monterrey, Nuevo León, México.

₹ Doctorando en Bioética. Jefe de Departamento de Medicina Familiar del Hospital «José Eleuterio González». Universidad Autónoma de Nuevo León, Monterrey, Nuevo León, México.

${ }^{\S}$ Doctora en Bioética. Profesora del

Departamento de Medicina Familiar del Hospital «José Eleuterio González». Universidad Autónoma de Nuevo León, Monterrey, Nuevo León, México.

" Médica Interna de Pregrado. Líder del Grupo Estudiantil de Bioética e Investigación en Medicina en Hospital «José Eleuterio González» y Facultad de Medicina de la Universidad Autónoma de Nuevo León, Monterrey, Nuevo León, México.

"Doctorante en Criminología. Presidente de Colegio de Peritos del Norte AC, Monterrey, Nuevo León México.

Correspondencia: $Y O A$, yeyetsy_10@hotmail.com, yeyeazuara@gmail.com Conflicto de intereses: Ninguno. 


\section{INTRODUCCIÓN}

La bioética es una disciplina que surgió relativamente hace poco. El término bioética fue usado por primera vez en 1927 por Fritz Jahr, pero no fue sino hasta 1970 que Van Rensselaer Potter lo acuñó en su escrito La ciencia de la supervivencia, y lo reafirmó en el año 1971 en su libro Bioética: Un puente hacia el futuro, en el cual la describe como «un puente entre la ciencia y el humanismo»?

Si bien hubo diferentes iniciativas organizacionales para el estudio de los valores y salud, o sobre la ética en medicina, fue en la década de los setenta en Estados Unidos donde cobró mayor rigor esta disciplina con el surgimiento del Hastings Center en 1969 en Nueva York, el cual fue iniciado por Daniel Callahan, y el Kennedy Institute of Ethics en 1971 en la Universidad de Georgetown, en Washington DC, el cual fue fundado por André Hellegers. Este centro actualmente es un referente literario en bioética, ya que por la interdisciplinariedad de sus exponentes, se le otorgó una mayor profundidad en su estudio. Es justo ahí, a través de la coordinación de Warren Reich, que en 1978 publicaron la primera Enciclopedia de bioética, consistente en cuatro volúmenes. En ésta, se establece la definición de bioética que hasta hoy continúa siendo la más aceptada:

«La bioética consiste en el estudio sistemático y científico de la conducta humana en el ámbito de las ciencias de la vida y de la salud, analizados a la luz de los valores y de los principios morales».2

Se ha vuelto ineludible incluir la bioética en el currículo de todos los programas formativos para el personal de salud, ya que ésta permea la visión interdisciplinaria tan necesaria para el abordaje de los problemas que atañen al ejercicio médico, incluso para los más complejos; además, la bioética favorece el cambio adecuado en los comportamientos

Citar como: Ordóñez AY, Gutiérrez HRF, Gómez GC, Cisneros GMS, González VDR. Conceptos y códigos clave para médicos sin formación bioética: revisión bibliográfica. Rev CONAMED. 2020; 25(3): 129-145. https://dx.doi.org/10.35366/95985

Financiamiento: Ninguno.

Recibido: 26/05/2020. Aceptado: 26/06/2020. y actitudes que obligan a médicos y a otros integrantes del equipo de salud a promover una relación médico-paciente, y entre los mismos profesionales a una relación más saludable, lo cual genera cambios positivos en los modelos de salud, comenzando con una toma de decisiones asertivas. Se dice incluso que si queremos recuperar el buen hacer de los profesionales de la salud, hay que empezar por darle valor a la enseñanza en lo referente al humanitarismo y, en el caso de la medicina, educar en principios y valores desde el punto de vista de la bioética., ${ }^{1,3}$

\section{Tabla 1: Declaración de Ginebra. ${ }^{4}$}

Promesa del médico

Como miembro de la profesión médica:

Prometo solemnemente dedicar mi vida al servicio de la humanidad

Velar ante todo por la salud y el bienestar de mis pacientes

Respetar la autonomía y dignidad de mis pacientes Velar con el máximo respeto por la vida humana No permitir que consideraciones de edad, enfermedad o incapacidad, credo, origen étnico, sexo, nacionalidad, afiliación política, raza, orientación sexual, clase social o cualquier otro factor se interpongan entre mis deberes y mis pacientes

Guardar y respetar los secretos que se me hayan confiado, incluso después del fallecimiento de mis pacientes

Ejercer mi profesión con conciencia y dignidad, conforme a la buena práctica médica

Promover el honor y las nobles tradiciones de la profesión médica

Otorgar a mis maestros, colegas y estudiantes el respeto y la gratitud que merecen

Compartir mis conocimientos médicos en beneficio del paciente y del avance de la salud

Cuidar mi propia salud, bienestar y capacidades para prestar una atención médica del más alto nivel

No emplear mis conocimientos médicos para violar los derechos humanos y las libertades ciudadanas, ni siquiera bajo amenaza

Hago esta promesa solemne y libremente, empeñando mi palabra de honor 
Tabla 2: Código Internacional de Ética Médica.'

Deberes de los médicos en general

El médico debe

- Siempre aplicar su opinión profesional independiente y mantener el más alto nivel de conducta profesional

- Respetar el derecho del paciente competente a aceptar o rechazar un tratamiento

- Permitir que su opinión sea influenciada por beneficio personal o discriminación injusta

- Dedicarse a proporcionar un servicio médico competente, con plena independencia profesional y moral, con compasión y respeto por la dignidad humana

- Tratar con honestidad a pacientes y colegas, e informar a las autoridades apropiadas sobre los médicos que practiquen en forma antiética e incompetente o a los que incurran en fraude o engaño

- Respetar los derechos y preferencias del paciente, de los colegas y de otros profesionales de la salud

- Reconocer su importante función en la educación de la opinión pública, pero debe obrar con la debida cautela al divulgar descubrimientos o nuevas técnicas o tratamientos a través de canales no profesionales

- Certificar sólo lo que ha verificado personalmente

- Esforzarse por utilizar los recursos de salud de la mejor manera para beneficio de los pacientes y su comunidad

- Buscar atención y cuidados apropiados si sufre una enfermedad mental o física

- Respetar los códigos de ética locales y nacionales

- Recordar siempre la obligación de respetar la vida humana

- Considerar lo mejor para el paciente cuando preste atención médica

- A sus pacientes toda su lealtad y todos los recursos científicos disponibles para ellos. Cuando un examen o tratamiento sobrepase su capacidad, el médico debe consultar o derivar a otro médico calificado en la materia

- Respetar el derecho del paciente a la confidencialidad. Es ético revelar información confidencial cuando el paciente otorga su consentimiento o cuando existe una amenaza real e inminente de daño para el paciente u otros y esta amenaza sólo puede eliminarse con la violación del secreto

- Prestar atención de urgencia como deber humanitario, a menos que esté seguro de que otros médicos pueden y quieren prestar dicha atención

- En situaciones cuando represente a terceros, asegurarse de que el paciente conozca cabalmente dicha situación

El médico no debe

- Permitir que su opinión sea influenciada por beneficio personal o discriminación injusta

- Recibir ningún beneficio financiero ni otros incentivos sólo por derivar pacientes o prescribir productos específicos

- Tener relaciones sexuales con sus pacientes actuales, ni ninguna otra relación abusiva o de explotación

\section{Deberes de los médicos hacia los colegas}

El médico debe

- Comportarse hacia sus colegas como él desearía que ellos se comportasen con él

- Cuando sea médicamente necesario, comunicarse con los colegas que atienden al mismo paciente. Esta comunicación debe respetar la confidencialidad del paciente y limitarse a la información necesaria

El médico no debe

- Dañar la relación médico-paciente de los colegas a fin de atraer pacientes 
Sin embargo, la inclusión de la bioética en las aulas universitarias es reciente. Específicamente, en México, la incursión de la bioética inició con la creación de organizaciones, para posteriormente integrarse en los currículos universitarios; en un inicio, en los estudios de postgrado, y luego en los de pregrado. Sin embargo, en el país son pocos los programas académicos de la licenciatura en medicina que la incluyen en su malla curricular. Específicamente, en la Facultad de Medicina de la Universidad Autónoma de Nuevo León (UANL) se imparte a través de la coordinación del Departamento de Medicina Familiar y desde hace 20 años en el postgrado. En 2017, inició la formación en el pregrado, formando en bioética a alrededor de mil estudiantes de medicina por año. Pero no todas las universidades cuentan con esta fortuna, ya que no incluyen a la bioética en el programa de estudios. Es por ello que actualmente gran cantidad de médicos mexicanos que ejercen o se encuentran en formación desconocen esta disciplina; esta situación condiciona desde la ignorancia una mala relación médico-paciente o entre los mismos profesionales a una falta de formación para resolución de dilemas médicos, e inclusive, los conduce a demandas legales por imprudencia, impericia o negligencia en el acto médico; todas éstas se podrían percibir como situaciones de mala praxis. . $^{5-7}$

El objetivo del presente trabajo es identificar los códigos bioéticos relacionados con la medicina desde la ética y legalidad para el ejercicio cabal de la profesión médica actual en México.

\section{MATERIAL Y MÉTODOS}

Con el propósito de compilar de forma clara y estructurada la información respecto al tema, se realizó una búsqueda bibliográfica a través de diferentes fuentes de información, buscadores y bases de datos, entre ellas: Google Scholar, Medline, Pubmed y SciELO. Se incluyeron en el presente trabajo las revisiones del tema, los artículos de investigación y los capítulos de libros; posteriormente, se hizo un análisis de contenido y selección de literatura por un grupo de expertos en bioética, lo que permitió elaborar un documento guía para el profesional de la salud, cuyo fin permita conocery atender de forma oportuna los aspectos relacionados con la bioética en la atención médica.

\section{RESULTADOS}

Actualmente, la bioética es activa y se refiere a hacer ciencia con conciencia humanitaria. Para su comprensión resulta, por tanto, diferenciarla de los conceptos de moral y ética. ${ }^{8,9}$

La moral es el conjunto de normas, reglas, creencias o costumbres que caracterizan a un individuo, grupo o comunidad, las cuales guían y determinan sus pautas conductuales en los actos de la vida cotidiana. La conducta se describe y el individuo o grupo decide si es bueno o malo, acorde con lo ya establecido. La moral es a priori al acto, puesto que la persona o grupo tiene una moral que determina su actuar. 2,8-10

La ética es la rama de la filosofía que estudia lo correcto o incorrecto del comportamiento humano. Proviene del latín tardío ethicus y del griego ethicós derivado de ethos, que significa «manera de ser, carácter», que de forma repetida dará lugar al hábito, que al establecerse originará el comportamiento. La ética es la reflexión crítica y racional del «ethos», de la conducta, basándose en fundamentos y pareciera dependiente del contexto. Como ciencia, es un conjunto de conocimientos derivados de la investigación de la conducta humana al tratar de explicar las reglas morales de manera racional, fundamentada teórica y científicamente. La ética es a posteriori al acto, puesto que del análisis sobre el acto surge la reflexión de la moral que lo origina, para luego construir las bases teóricas del porqué algo es bueno o malo.

La ética obliga a la reflexión sobre nuestras creencias, prácticas y juicios morales, ya que no sólo estudia lo que se hace, sino también lo que deja de hacerse por negligencia, mala planeación y falta de previsión. 2,8-10

En el caso de la ética profesional, ésta exige la excelencia y busca la formación del carácter y el desarrollo de competencias cognitivas, técnicas sociales y éticas en un área específica del conocimiento. Por su parte, la deontología hace referencia al cumplimiento estricto de los deberes y apunta al conocimiento de lo que es justo y conveniente para el ejercicio de una determinada profesión, razón por la cual forma parte de la ética profesional. Los códigos deontológicos son un conjunto de principios y reglas que han de guiar una conducta profesional, hablan de los deberes profesionales y 


\section{Tabla 3: Código de Conducta de la Secretaría de Salud."}

Capítulo

\section{Introducción \\ II. Objetivo \\ III. Misión y visión de la \\ Secretaría de Salud \\ IV. Ámbito de aplicación}

V. Marco Jurídico

VI. Principios y valores del código de Ética de los Servidores Públicos del Gobierno Federal

VII. Reglas de integridad

VIII. Protocolo de actuación en la atención de presuntos actos de discriminación

IX. Valores específicos de la Secretaría de Salud
Este código deberá ser aplicado por todas las personas que laboren o presten sus servicios en la Secretaría de Salud y sus órganos desconcentrados, independientemente del contrato al que estén sujetos

1. Legalidad
2. Honradez
3. Lealtad
4. Imparcialidad
5. Eficiencia
1. Interés público
2. Respeto
3. Respeto a los Derechos Humanos
4. Igualdad y no discriminación
5. Equidad de género
6. Entorno Cultural y Ecológico
7. Integridad
8. Cooperación
9. Liderazgo
10. Transparencia
11. Rendición de cuentas

El protocolo tiene como objetivo el establecer acciones para prevenir y atender los casos de discriminación; todo servidor público de la Secretaría de Salud debe vigilar que su entorno laboral sea libre de cualquier forma de discriminación

Conocimiento y observancia de la normativa aplicable Debido ejercicio del servicio público

Equidad

Honestidad
No discriminación

Rectitud

Responsabilidad

Solidaridad

Sustentabilidad

Tolerancia

X. Glosario

tienen como finalidad el garantizar la buena práctica de la profesión. ${ }^{12}$

La ética médica hace referencia a los requerimientos que tiene el acto médico de principios éticos y morales fundamentales, de conocimientos científicos actualizados, de habilidades refinadas y de acciones responsables.
La deontología sólo incluye normas y deberes de un área, por lo tanto, la deontología médica establece qué deben y qué no deben de hacer los médicos. Esta última se refiere a las reglasy normas que regulan la relación del médico con los enfermos, con la sociedad, con los colegas de profesión, con los otros profesionales, con la administración, etcétera. ${ }^{13}$ 
Aun cuando la conducta bioética se encuentra plasmada en diferentes medios impresos y electrónicos, es difícil integrar la información disponible para el médico, y aún más para el estudiante de medicina, por lo que a continuación se describen los principales códigos relacionados con el ejercicio cabal de la profesión médica desde la perspectiva bioética.

A nivel internacional, la Asociación Médica Mundial (AMM) es aquélla que se ha dado el objetivo de formular y promover las normas relacionadas con la deontología médica. Entre los documentos más importantes sobre políticas de ética, destacan:

- La Declaración de Ginebra (Juramento Hipocrático moderno).

- La Declaración de Helsinki (normas de investigación médica en seres humanos).

- La Declaración de Tokio (normas dirigidas a médicos para prevenir la tortura).

- La Declaración de Taipéi (normas sobre investigación de bases de datos, datos masivos y biobancos).

En este trabajo, nos referimos a la prestación de servicios de salud a la atención médica solamente, razón por la cual resumimos la Declaración de Ginebra de 2017 en la Tabla 7.,14

Acorde con la AMM, el juramento no debe ser leído solo, sino que debe enunciarse junto con las políticas detalladas en el Código Internacional de Ética Médica. ${ }^{15}$ Este código fue adoptado por la Tercera Asamblea General de la AMM en 1949, y ha sido revisado en 1968, 1983 y 2006. El Código Internacional de Ética Médica es el conjunto de disposiciones inspiradas en principios éticos universales, que regula la conducta médica en el ejercicio de su profesión y en su relación con la sociedad. Éste se compone por:

- Doce deberes de los médicos en general, los cuales establecen cómo debe ser su conducta y actitud profesional.

- Siete deberes de los médicos hacia los enfermos, hacia sus pacientes o a la gente que tratará.

- Tres deberes de los médicos hacia los colegas.

Todos estos deberes se describen en la Tabla 2.
A nivel nacional, el Código Ético que rige el comportamiento profesional del personal médico en México es el Código de Conducta de la Secretaría de Salud (última revisión en febrero de 2018), el cual está basado en el Código de Ética de los servidores públicos proporcionado por el Gobierno Federal. En este código, se establecen los principios constitucionales que son la base del trabajo del profesional, los valores presentes en un buen médico y las reglas de integridad para el ejercicio de la función pública (Tabla 3)!"

En consideración con el Movimiento Bioético Universal, en el campo de la responsabilidad de los profesionales, se presentó el Código de Bioética para el Personal de Salud (2002), que representa una guía de conducta en el ejercicio profesional, con el fin de resolver las diferencias entre los actores que intervienen en los acontecimientos relacionados con la medicina y la salud (Tabla 4). ${ }^{10,16}$

En México, si bien es normativo seguir los códigos internacionales, es un deber ejercer en apego a la Ley General de la Salud, la cual es la ley que reglamenta el derecho a la protección de la salud que tiene todo mexicano. Este derecho se adicionó al Artículo 4. ${ }^{\circ}$ de la Constitución Política de los Estados Unidos Mexicanos durante el sexenio de Miguel de la Madrid, publicándose la noticia en el Diario Oficial de la Federación el 3 de febrero de 1983, habiéndose así elevado este derecho social a la máxima jerarquía. Fue el 26 de diciembre de 1983 cuando el Congreso de la Unión aprobó la Ley General de Salud y se publicó el 7 de febrero de 1984 en el Diario Oficial de la Federación, entrando en vigor el primero de julio del mismo año. ${ }^{17}$

La Ley General de Salud consta de 18 capítulos y 482 Artículos que buscan garantizar la salud de la población mexicana. Desde su publicación original, ha sido reformada constantemente hasta llegar a vislumbrarse su más reciente versión el 12 de julio de 2018. A pesar de ser gratuita en medios electrónicos y accesible en medios impresos, es poco conocida. En la Tabla 5 se presenta su estructura para que sirva como guía para los profesionales de la salud.

Las Normas Oficiales Mexicanas (NOM) son regulaciones técnicas de observancia obligatoria expedidas por las dependencias competentes, 
Tabla 4: Código de Bioética para el personal de Salud.16

Capítulo

I. Principios básicos

1. Toda persona tiene, en principio, derecho a la protección de su salud. Los habitantes de la República Mexicana y los mexicanos que habiten en el extranjero deben gozar de este mismo derecho. El personal de salud está obligado a buscar los medios para que se cumplan los deberes de equidad y justicia, paradigma prioritario de la bioética

2. Las acciones de atención a la salud proporcionadas por el personal profesional y técnico deben ser aplicadas en beneficio de la población en un marco científico y humanitario

3. El personal de salud debe atender a todos los que demanden sus servicios sin distinción de edad, sexo, nacionalidad, religión, ideología política, posición socioeconómica; sin escatimar tiempo, ni espacio, respetando siempre la dignidad y derechos humanos

4. El personal de salud tiene el compromiso de salvaguardar el prestigio de las profesiones que participen en las actividades de la atención médica, con atributos de honradez, capacidad y eficiencia

5. Es necesario afirmar que el respeto a la dignidad humana se basa en el principio fundamental de que los seres humanos poseen igual valor

II. Conducta bioética en la prestación de servicios de salud

III. El enfermo como centro de interés de la bioética
6. La población sana o enferma merece recibir del personal que presta atención a la salud lo mejor de sus conocimientos y destrezas en su beneficio, con interés genuino de mantener su salud y bienestar, y un trato amable, respetuoso, prudente y tolerante

7. El personal de salud tiene la obligación de participar en el ámbito de su competencia en actividades que contribuyan al beneficio de la salud de la comunidad, así como en la atención médica en casos de emergencia y desastres

8. La actitud del equipo de salud debe mantener e incrementar la confianza de los individuos a su cargo, especialmente de los pacientes y personal que los asiste, puesto que el humanitarismo con el que se preste la atención al enfermo contribuye directamente a su estabilización, recuperación o rehabilitación

9. Las instituciones de salud, conforme con sus posibilidades, deberán asignar a cada médico u otros prestadores de servicios el número adecuado de personas de acuerdo con la complejidad de su atención de salud, considerando que la sobrecarga de trabajo y la fatiga son poco propicias para otorgar servicios de alta calidad

10. Con base en las políticas de la institución y en casos urgentes, si el paciente es incapaz de tomar una decisión para su diagnóstico y tratamiento y en ausencia de su representante legal, el personal de salud podrá aplicar las medidas que considere pertinentes y sean profesionalmente aceptadas

11. Es deber de los médicos y de las instituciones de salud realizar el seguimiento de los pacientes durante una enfermedad crónica o aguda y no abandonarlos mientras dure su enfermedad o se rehabilite

12. El prestador de servicios de salud tiene la responsabilidad de sus enfermos a los que debe estar dispuesto a atender. Tratará de resolver cualquier diferencia o falta de afinidad con algún paciente, reconociéndole su autonomía, y presentándole alternativas en su tratamiento y, sólo cuando lo amerite por no contar con los medios adecuados, lo derivará a otro colega o nivel para su atención

13. Los enfermos son personas que padecen trastornos que alteran su estado físico, emocional y social, lo que los hace particularmente vulnerables, por lo que deben recibir un trato de consideración, amabilidad y respeto. Esto mismo es aplicable a sus familiares, que comúnmente presentan angustias e inquietudes 
Continúa Tabla 4: Código de Bioética para el personal de Salud. ${ }^{16}$

Capítulo

IV. Potestades

bioéticas del

personal de salud
14. El paciente, o su representante legal, debe ser informado por el médico tratante, con sentido humano, sobre su enfermedad, las alternativas para su tratamiento, las probables complicaciones y cómo evitarlas. Esto se expresará con lenguaje claro y veraz, de forma que sea inteligible para el enfermo, lo que le permitirá ser partícipe en el manejo de su propia enfermedad

15. La información que pudiera producir inquietud o daño psicológico al paciente debe proporcionársele con la prudencia debida, en el momento oportuno, en la extensión que el paciente determine y del modo que el equipo de salud considere más adecuada a las circunstancias para el beneficio del enfermo

16. La información de la enfermedad de un paciente debe guardarse con confidencialidad, salvo cuando la normatividad epidemiológica determine su notificación o que sea requerida por orden judicial o instancias legales que correspondan

17. La responsabilidad del manejo confidencial de los expedientes clínicos, escritos o en registros electrónicos, recae en todas aquellas personas que tienen acceso a esa información

18. Todo paciente tiene derecho a que se le respete su privacidad cuando proporciona información al prestador de servicios, cuando se comunica con sus familiares o cuando su pudor así lo exige. Las instituciones de salud deben contar con los medios para que este precepto se cumpla y el personal cuidará que no se quebrante

19. El paciente estará informado de los nombres y cargos del personal de salud que lo trata, sobre la probabilidad de ser atendido por otras personas y dónde acudir a presentar alguna queja

20. El enfermo, en cualquier momento de su vida, puede expresar por escrito su voluntad anticipada para evitar que si sus condiciones no le permiten tomar decisiones o expresarlas, se le apliquen medidas extraordinarias por su alta complejidad o desproporcionadas en relación con los resultados esperados, con las que se intente prolongarle la vida, si no existen probabilidades razonables de regresar a una vida física, psicológica o socialmente aceptable

21. El personal de salud debe realizar, en coordinación con las autoridades competentes, las medidas preventivas pertinentes que sean estrictamente necesarias para el bien de la población y adicionales a la normatividad establecida, tales como saneamiento básico, agua potable, eliminación sanitaria de excretas, control de fauna nociva, inocuidad de alimentos, vacunaciones, aislamiento de pacientes infecto-contagiosos o agresivos y otras acciones que considere convenientes en provecho colectivo

22. Las instituciones de salud, de acuerdo con su nivel de atención, deberán proporcionar con oportunidad en calidad y cantidad suficientes, el equipo, instrumental, materiales de consumo y medicamentos que requiere el personal para proporcionar la adecuada atención

23. Los prestadores de servicios de salud deben actualizar y certificar sus conocimientos y destrezas para otorgar a sus pacientes la mejor atención posible. Las instituciones oficiales o privadas donde laboran estarán comprometidas a realizar actividades académicas y proporcionar los medios de aprendizaje como libros, revistas, programas electrónicos y otros, de acuerdo con los servicios que otorguen

24. Es responsabilidad de los directivos y docentes de las instituciones académicas que preparan profesionales y técnicos en las ramas del cuidado de la salud, que la capacitación sea del más alto nivel, con el apoyo de las instituciones de salud 
Continúa Tabla 4: Código de Bioética para el personal de Salud. ${ }^{16}$

Capítulo

V. Responsabilidades de los individuos sanos y de los pacientes
25. Los miembros del equipo de salud tienen derecho a percibir una remuneración adecuada por su trabajo, en relación con su capacidad profesional y el número de horas que laboran en la institución, o según el acuerdo previo que hayan tenido con un paciente privado. Esta remuneración debe tener relación con las costumbres y el nivel socioeconómico de la población, sin caer en abusos y con un espíritu de condescendencia y compasión por los pacientes con escasos recursos

26. El personal de salud puede expresar a las autoridades directivas y administrativas de la institución con toda libertad y respeto las ideas que considere pueden beneficiar a la institución y a los pacientes. Con este criterio, si un miembro del equipo de salud considera que algún reglamento o alguna ley son injustos, pondrá su empeño en que las instancias modifiquen esas disposiciones por los canales debidos

27. El personal de salud deberá contar con las instalaciones, equipos, instrumentos y materiales para el desempeño de sus actividades con las medidas de higiene, protección y seguridad. De no contar con ellos, podrá rehusarse a aplicar los procedimientos diagnósticos y terapéuticos, sin contravenir los principios bioéticos, manifestándolo por escrito y procurando que el paciente sea referido a otra unidad médica

28. El personal de salud podrá rehusarse a aplicar medidas diagnósticas y terapéuticas que a su juicio pongan en riesgo la vida, la función de los pacientes o su descendencia, bien sea a petición de los propios pacientes, de sus superiores jerárquicos o autoridades institucionales, cuando se oponga a la práctica médica comúnmente aceptada, a los principios bioéticos, a sus capacidades profesionales o a razones de objeción de conciencia

29. La población debe informarse del cuidado de la salud y procurar desarrollar actitudes y conductas sin riesgo para su salud y la de la comunidad

30. Los pacientes o representantes legales tienen la responsabilidad de informar con veracidad al personal de salud, así como de manifestar las dudas y preocupaciones relacionadas con su enfermedad

31. El consentimiento válidamente informado del paciente es la más sólida expresión de su autonomía, por lo que deben plantearse las alternativas de atención para la toma de sus decisiones. El consentimiento informado establece su autodeterminación para aceptar o rechazar métodos de diagnóstico, tratamiento y cuidados generales, excepto cuando de existir rechazo suponga riesgos de salud pública

32. El paciente tiene derecho a que se respete su decisión de aceptar o no cualquier procedimiento de diagnóstico o tratamiento. Este precepto debe aplicarse a los niños, ancianos, o pacientes con trastornos mentales leves, si su estado de madurez y lucidez es apropiado para tomar la decisión correspondiente. Su aceptación en estos casos debe ser ratificada por los padres, tutores o responsables

33. El paciente, después de haber recibido la información detallada y aceptar las prescripciones médicas, está comprometido a cumplirlas con precisión. Tiene el deber de informar al personal que lo atiende cuando por cualquier motivo ha dejado de cumplir las indicaciones

34. El enfermo que sabe que tiene una enfermedad contagiosa adquiere, al saberlo, la obligación de evitar por todos los medios que otras personas enfermen por su contacto con él 
Continúa Tabla 4: Código de Bioética para el personal de Salud. ${ }^{16}$

Capítulo

VI. Relaciones interpersonales del equipo de salud

VII. Bioética en la investigación en salud

VIII. Temas bioéticos específicos
35. La persona que se sabe portadora de información genética adversa susceptible de transmitirse a su descendencia adquiere el compromiso de seguir el consejo genético que se le proporcione, según el caso y asume completamente la responsabilidad de sus actos

36. La capacidad de los miembros del equipo de salud, su colaboración, armonía y disposición de cooperar con los demás en beneficio de los pacientes y de la comunidad, constituyen las bases de la conducta bioética y el sustento de la calidad de los servicios de salud

37. Es deber del equipo de salud transmitir sus conocimientos a sus colegas, alumnos, pacientes y a la sociedad en general. La enseñanza y la información sobre los medios para proteger la salud han de ser consideradas como unas de las obligaciones más relevantes de los prestadores de salud

38. Las dicotomías o dividendos económicos entre el personal de salud por la derivación de pacientes a otros médicos, laboratorios y gabinetes, o por la prescripción de tratamientos son actos inaceptables, contrarios a la dignidad y a la ética de los profesionales y técnicos de la salud y al bienestar del paciente

39. El personal de salud puede señalar las fallas o desacuerdos que a su juicio se presenten con otros profesionales y técnicos, siempre con respeto, procurando no dañar el prestigio de algún colega o colaborador al hacerlo

40. El beneficio que los pacientes y la comunidad obtienen de las actividades médicas se basa en el progreso de las ciencias de la salud que se fundamenta en la investigación. Participar en las investigaciones es una obligación moral del personal, fuente de su propio desarrollo

41. Las investigaciones básicas, clínicas u operacionales en las que interviene el equipo de salud deben apegarse a la legislación sanitaria y ser aprobadas por los Comités creados ex profeso en las instituciones donde laboran, previo análisis de los protocolos diseñados por los participantes en el estudio

42. Los investigadores recabarán el consentimiento informado por escrito de las personas sujetas a investigación o en su caso, de sus apoderados o tutores. El consentimiento se obtendrá con la clara descripción del estudio, de sus riesgos, beneficios y el planteamiento del derecho del paciente a retirarse de la investigación cuando así lo desee, sin que ello afecte en modo alguno su ulterior atención médica

43. Las investigaciones promovidas por la industria farmacéutica u otros productores de insumos para la salud deben sujetarse estrictamente a las normas de cualquier investigación en humanos y asegurar a las personas involucradas el máximo beneficio durante la investigación, cumpliendo además con la normatividad y justificando su realización

44. Particular cuidado se tendrá en no emplear en una investigación como grupo de estudio o grupo testigo a personas que estén dispuestas a aceptar su colaboración sólo por su condición de subordinados o cautivos, o por el ofrecimiento de incentivos económicos

45. Los animales de experimentación se emplearán sólo cuando resulten indispensables y no haya pruebas alternativas confiables. En todo caso, se cumplirán las normas de protección y respeto para evitar sufrimiento innecesario

46. La donación de órganos y tejidos para trasplantes debe ser promovida por el personal de salud y por la población en general 
Continúa Tabla 4: Código de Bioética para el personal de Salud. ${ }^{16}$

Capítulo

47. La interrupción de un embarazo no es jamás un acto deseable. La ley ha establecido cuáles pueden ser los casos en que no está penalizado, entre los que se encuentran los que ponen en riesgo la vida o la salud de la mujer gestante, que justificarían esta intervención, sin que se pueda obligar a participar al personal que manifieste cualquier objeción

48. Es éticamente adecuado utilizar los avances de la ciencia para ayudar a las parejas infértiles a tener hijos mediante procedimientos de reproducción asistida que tengan alto grado de seguridad para la salud de la madre y practicados por personas expertas en instalaciones que cuenten con los equipos y demás medios necesarios

49. La investigación genética para conocer los fenómenos biológicos de la herencia y que pueden favorecer la reproducción humana para la aplicación con fines terapéuticos debe preservar en sus métodos la dignidad y los derechos humanos

50. Al paciente terminal se le aplicarán los procedimientos más actuales de la medicina paliativa para aliviar en lo posible sus sufrimientos físicos y psicológicos, aunque esto pudiera acortar su vida sin que ello implique provocar su muerte

51. El personal de salud ejercerá la influencia moral que tiene ante la sociedad y ante las autoridades para promover las medidas de preservación de los sistemas ecológicos, la limpieza del agua, de los alimentos y de los factores que protejan la salud humana y la biodiversidad

que tienen como finalidad establecer las características que deben reunir los procesos o servicios cuando éstos puedan constituir un riesgo para la seguridad de las personas o dañar la salud humana; así como aquellas relativas a la terminología y las que se refieran a su cumplimiento y aplicación. Las NOM en materia de Prevención y Promoción de la Salud, una vez aprobadas por el Comité Consultivo Nacional de Normalización de Prevención y Control de Enfermedades (CCNNPCE), son expedidas y publicadas en el Diario Oficial de la Federación y, por tratarse de materia sanitaria, entran en vigor al día siguiente de su publicación. Estas normas deben ser revisadas cada cinco años a partir de su entrada en vigor. El CCNNPCE deberá de analizar y, en su caso, realizar un estudio de cada NOM, cuando su periodo venza en el transcurso del año inmediato anterior, y como conclusión de dicha revisión y/o estudio, podrá decidir la modificación, cancelación o ratificación de éstas. Se presentan en la Tabla 6 algunas NOM de importancia (por las necesidades de atención de acuerdo con la frecuencia) para el personal de la salud. ${ }^{18}$
Además, en la Tabla 7, presentamos las Recomendaciones Generales para Mejorar la Práctica de la Medicina. Éstas se derivaron del Consenso de la Primera Reunión Nacional de Comisiones Estatales de Arbitraje Médico, convocada por la CONAMED, en la cual el pleno consideró que, siguiendo estas recomendaciones, se mejorará la relación médico-paciente y se garantizará una atención médica de calidad a favor de la salud de la población mexicana. ${ }^{19}$

Los códigos antes mencionados son para los profesionales de la salud; sin embargo, no existe algún código formal para el médico en formación, es decir, para los estudiantes de medicina en México. Razón por la cual recomendamos aplicar la Guía de Recomendaciones Éticas para el Estudiante de Medicina, propuesta por la Comisión de Bioética del Consejo Estatal de Estudiantes de Medicina (CEEM) en España, para dar un acercamiento a la actitud bioética desde la formación. En este documento, se describen las cualidades y actitudes esperadas de un estudiante de medicina en su lugar de formación y ante los pacientes y profesionales; además, ofrece un capítulo para la toma de decisiones 
Tabla 5: Ley General de la Salud. ${ }^{17}$

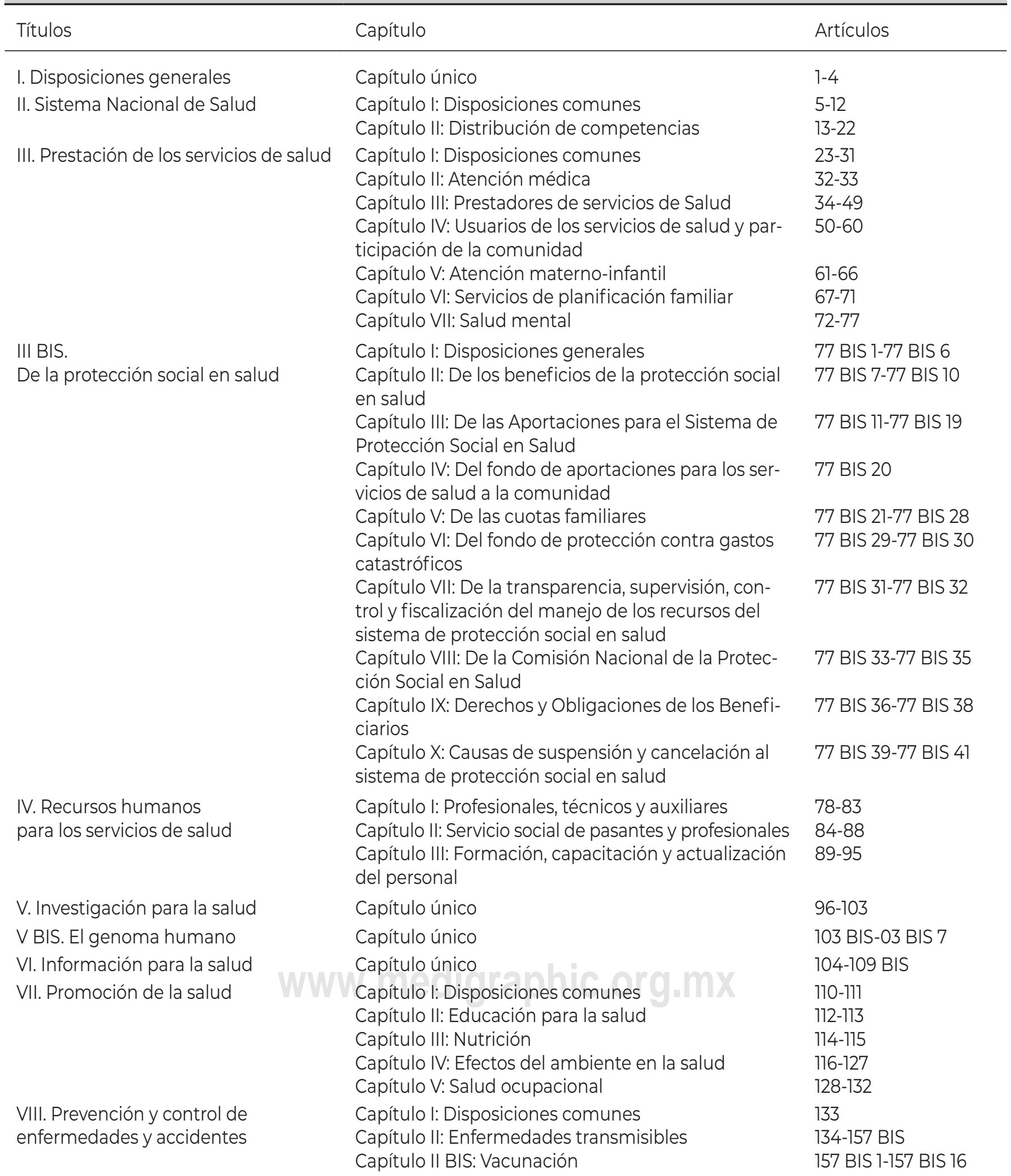


Continúa Tabla 5: Ley General de la Salud. ${ }^{17}$

\begin{tabular}{l} 
Títulos \\
\hline VIII BIS. De los cuidados paliativos a \\
los enfermos en situación terminal \\
IX. Asistencia social, prevención de la \\
discapacidad y rehabilitación de las \\
personas con discapacidad \\
X. Acción extraordinaria en materia \\
de salubridad general \\
XI. Programas contra las adicciones
\end{tabular}

XII. Control sanitario de productos y servicios, y de su importación y exportación
XIII. Publicidad
Capítulo

Capítulo III: Enfermedades no transmisibles Capítulo III BIS: Del Registro Nacional de Cáncer Capítulo IV: Accidentes

Capítulo I: Disposiciones comunes

Capítulo II: De los derechos de los enfermos en situación terminal

Capítulo III: De las facultades y obligaciones de las instituciones de salud

Capítulo IV: De los derechos, facultades y obligaciones de los médicos y personal sanitario

Capítulo único

Artículos

158-161

$161 \mathrm{BIS}$

162-166

166 BIS-166 BIS 2

166 BIS 3-166 Bis 12

166 BIS 13

166 BIS 14-166 BIS 21

$167-180$

Capítulo único

181-184

Capítulo I: Consejo Nacional contra las Adicciones Capítulo II: Programa para la prevención, reducción y tratamiento del uso nocivo del alcohol, la atención del alcoholismo y la prevención de enfermedades derivadas del mismo

Capítulo II BIS: Protección de la salud de terceros y de la sociedad frente al uso nocivo del alcohol Capítulo III: Programa contra el tabaquismo Capítulo IV: Programa contra la farmacodependencia

Capítulo I: Disposiciones Comunes

Capítulo II: Alimentos y bebidas no alcohólicas

Capítulo III: Bebidas alcohólicas

Capítulo IV: Medicamentos

Capítulo V: Estupefacientes

Capítulo Vl: Substancias Psicotrópicas

Capítulo VII: Establecimientos destinados al proceso de medicamentos

Capítulo VIII: Equipos médicos, prótesis, ortesis, ayudas funcionales, agentes de diagnóstico, insumos de uso odontológico, materiales quirúrgicos, de curación y productos higiénicos Capítulo IX: Productos cosméticos

Capítulo IX BIS: Ejercicio especializado de la cirugía Capítulo X: Productos de aseo Capítulo XI: Tabaco Capítulo XII: Plaguicidas, nutrientes vegetales y substancias tóxicas o peligrosas Capítulo XII BIS: Productos biotecnológicos Capítulo XIII: Importación y exportación

$184 \mathrm{BIS}$ 185-187

187 BIS-187 BIS 1

188-190 191-193 BIS

$194-214$

$215-216$

$217-220$

$221-233$

234-243

244-256

$257-261$

262-268 BIS 1

269-272

272 BIS-272 BIS 3

273-274

275-277 BIS

278-282

282 BIS-282 BIS 2

283-299

300-312 


\begin{tabular}{|c|c|c|}
\hline \multicolumn{3}{|c|}{ Continúa Tabla 5: Ley General de la Salud. ${ }^{17}$} \\
\hline Títulos & Capítulo & Artículos \\
\hline \multirow{7}{*}{$\begin{array}{l}\text { XIV. Donación, trasplantes, y pérdida } \\
\text { de la vida }\end{array}$} & Capítulo I: Disposiciones comunes & 313-319 \\
\hline & Capítulo II: Donación & $320-329 \mathrm{BIS}$ \\
\hline & Capítulo III: Trasplante & $330-339$ \\
\hline & Capítulo III BIS: Disposición de sangre, componen- & $340-342$ BIS 3 \\
\hline & $\begin{array}{l}\text { tes sanguineos, hemoderivados y celulas troncales } \\
\text { de seres humanos }\end{array}$ & \\
\hline & Capítulo IV: Pérdida de la vida & $343-345$ \\
\hline & Capítulo V: Cadáveres & 346-350 BIS 7 \\
\hline \multirow[t]{3}{*}{ XV. Sanidad Internacional } & Capítulo I: Disposiciones Comunes & $351-359$ \\
\hline & Capítulo II: Sanidad en materia de migración & $360-362$ \\
\hline & Capítulo III: Sanidad marítima, aérea y terrestre & $363-367$ \\
\hline \multirow[t]{3}{*}{ XVI. Autorizaciones y certificados } & Capítulo I: Autorizaciones & $368-379$ \\
\hline & Capítulo II: Revocación de autorizaciones sanitarias & $380-387$ \\
\hline & Capítulo III: Certificados & $388-392$ \\
\hline XVII. Vigilancia sanitaria & Capítulo Único & 393-401 BIS 2 \\
\hline \multirow{7}{*}{$\begin{array}{l}\text { XVIII. Medidas de seguridad, } \\
\text { sanciones y delitos }\end{array}$} & Capítulo I: Medidas de seguridad sanitaria & $402-415$ \\
\hline & Capítulo II: Sanciones administrativas & $416-427$ \\
\hline & $\begin{array}{l}\text { Capítulo III: Procedimiento para aplicar las medidas } \\
\text { de seguridad y sanciones }\end{array}$ & $428-437$ \\
\hline & Capítulo IV: Recurso de inconformidad & $438-450$ \\
\hline & Capítulo V: Prescripción & $451-454$ \\
\hline & Capítulo VI: Delitos & $455-472$ \\
\hline & $\begin{array}{l}\text { Capítulo VII: Delitos Contra la salud en su modalidad } \\
\text { de narcomenudeo }\end{array}$ & $473-482$ \\
\hline
\end{tabular}

difíciles basadas en los principios bioéticos. De esta manera, ofrece de manera sencilla un código deontológico básico para que el profesional en formación desarrolle una actitud bioética. ${ }^{20}$

Sin duda, la bioética es hacer ciencia con conciencia humanitaria, y para aquéllos que ejercemos la docencia en la actualidad, el reto es también a la inversa. Dicho de otro modo, crear conciencia humanitaria a través de la ciencia y la tecnología. Por eso, a través de este link: http://www.bioeticlass. com/2020/03/2-codigos-de-bioetica-para.html ponemos a tu disposición el acceso a los archivos con todos los códigos antes mencionados. ${ }^{21}$

\section{CONCLUSIONES}

Resulta de gran importancia conocer la bioética como «la disciplina que consiste en el estudio sistemático y científico de la conducta humana en el ámbito de las ciencias de la vida y de la salud, analizados a la luz de los valores y de los principios morales» ${ }^{2}$ y saberla diferenciar de los conceptos de moral y de ética. Esto permite reconocer que los conceptos y códigos clave relacionados con el ejercicio cabal de la medicina en la asistencia médica desde la perspectiva bioética son: Declaración de Ginebra, Código Internacional de ética Médica, Código de Conducta de la Secretaría de Salud, Código de Bioética para el Personal de Salud, Ley General de la Salud, Ias Normas Oficiales Mexicanas y las Recomendaciones generales para mejorar la práctica de la medicina de la CONAMED. Asimismo, recomendamos el uso de la Guía de Recomendaciones Éticas para el Estudiante de Medicina para los estudiantes en formación. 
Tabla 6: Normas Oficiales Mexicanas de importancia para el personal de salud. ${ }^{18}$

NOM-004-SSA3-2012

NOM-005-SSA2-1993

NOM-006-SSA2-2013

NOM-007-SSA2-1993

NOM-009-SSA2-2013

NOM-010-SSA2-2010

NOM-011-SSA2-2011

NOM-013-SSA2-2006

NOM-014-SSA2-1994

NOM-015-SSA2-2010

NOM-016-SSA2-2012

NOM-017-SSA2-2012

NOM-021-SSA2-1994

NOM-022-SSA2-2012

NOM-025-SSA2-1994

NOM-027-SSA2-2007

NOM-028-SSA2-2009

NOM-029-SSA2-1999

NOM-030-SSA2-2009

NOM-031-SSA2-1999

NOM-032-SSA2-2010

NOM-033-SSA2-2011

NOM-034-SSA2-2013

NOM-035-SSA2-2012

NOM-036-SSA2-2012

NOM-037-SSA2-2012

NOM-038-SSA2-2010

NOM-039-SSA2-2002

NOM-041-SSA2-2011

NOM-042-SSA2-2006

NOM-043-SSA2-2012

NOM-045-SSA2-2005

NOM-046-SSA2-2005

NOM-047-SSA2-2015
Del expediente clínico

De los servicios de planificación familiar

Para la prevención y control de la tuberculosis

Atención de la mujer durante el embarazo, parto y puerperio y del recién nacido. Criterios y procedimientos para la prestación del servicio

Promoción de la salud escolar

Para la prevención y control de la infección por virus de la inmunodeficiencia humana

Para la prevención y control de la rabia humana y en los perros y gatos

Para la prevención y control de enfermedades bucales

Para la prevención, detección, diagnóstico, tratamiento, control y vigilancia epidemiológica del cáncer cervicouterino

Para la prevención, tratamiento y control de la diabetes mellitus

Para la vigilancia, prevención, control, manejo y tratamiento del cólera

Para la vigilancia epidemiológica

Para la vigilancia, prevención y control del complejo teniosis/cisticercosis en el primer nivel de atención médica

Para la prevención y control de la brucelosis en el ser humano

Para la prestación de servicios de salud en unidades de atención integral hospitalaria médico-psiquiátrica

Para la prevención y control de la lepra

Para la prevención, tratamiento y control de las adicciones

Para la vigilancia epidemiológica, prevención y control de la leptospirosis en el humano

Para la prevención, detección, diagnóstico, tratamiento y control de la hipertensión arterial sistémica

Para la atención a la salud del niño

Para la vigilancia epidemiológica, prevención y control de las enfermedades transmitidas por vector

Para la vigilancia, prevención y control de la intoxicación por picadura de alacrán

Para la prevención y control de los defectos al nacimiento

Para la prevención y control de enfermedades en la perimenopausia y postmenopausia de la mujer. Criterios para brindar atención médica

Prevención y control de enfermedades. Aplicación de vacunas, toxoides, faboterápicos (sueros) e inmunoglobulinas en el humano

Para la prevención, tratamiento y control de las dislipidemias

Para la prevención, tratamiento y control de las enfermedades por deficiencia de yodo

Para la prevención y control de las infecciones de transmisión sexual

Para la prevención, diagnóstico, tratamiento, control y vigilancia epidemiológica del

cáncer de mama

Prevención y control de enfermedades. Especificaciones sanitarias para los centros de atención canina

Servicios básicos de salud. Promoción y educación para la salud en materia alimentaria. Criterios para brindar orientación.

Para la vigilancia epidemiológica, prevención y control de las infecciones nosocomiales Violencia familiar, sexual y contra las mujeres. Criterios para la prevención y atención Para la atención a la salud del Grupo Etario de 10 a 19 años de edad 
Tabla 7: Recomendaciones generales para mejorar la práctica de la medicina. ${ }^{19}$

1. Mantener una relación respetuosa con el paciente y su familia

2. Informar y obtener el válido consentimiento por escrito antes de realizar procedimientos con riesgo

3. Elaborar un expediente clínico completo

4. Actuar con bases científicas y apoyo clínico

5. Proceder sólo con facultad y conocimientos

6. Garantizar seguridad en las instalaciones y equipo

7. Atender a todo paciente en caso de urgencia y nunca abandonarlo
- Identificarse con el paciente y su familia

- Evitar malos tratos

- No demorar injustificadamente la atención

- No discriminar por ninguna razón al paciente

- Hablar con lenguaje entendible

- Ser tolerante, paciente y escuchar al enfermo y a su familia

- Mantener la confidencialidad

- Ser claro, no confundir al paciente en cuanto a su diagnóstico, pronóstico y no mentirle

- Ofrecer información clara, completa, veraz, oportuna y calificada

- Permitir la segunda opinión

- No presionar al paciente a decidir cuando no haya una urgencia real

- Solicitar el válido consentimiento informado (información amplia, sin coerción, ante un paciente competente y capacitado para aceptar o rehusar)

- Proporcionar resumen del expediente al paciente cuando lo solicite

- Por ningún motivo alterar el expediente

- Conservarlo por un mínimo de cinco años

- Firmar todas las notas

- Actuar según el Arte Médico, los conocimientos científicos y los recursos a su alcance

- Evitar la medicina defensiva, ya que no es ético

- Evitar prácticas inspiradas en charlatanería

- No simular tratamientos

- Evitar prescribir medicamentos de composición no conocida

- Evitar consultas por teléfono, radio, carta e internet

- Sólo actuar cuando se tiene la capacidad reconocida para hacerlo (Título o Diploma)

- Recurrir a otro compañero cuando el caso esté fuera de su capacidad o competencia

- No participar en prácticas delictivas como el aborto, eutanasia activa, falsos certificados médicos, ni retener pacientes por falta de pago y otras razones

- Disponer de libertad de prescripción

- No participar en prácticas con dicotomía

- Mantener una permanente actualización médica

- Conocer la capacidad instalada de la unidad de salud donde se pretende dar atención

- Probar el equipo que se podrá utilizar

- Referir a otra unidad al paciente cuando no se le garantice seguridad en las instalaciones

- Informar al paciente y su familia sobre la capacidad instalada de la unidad de salud

- Preferir, cuando sea posible, hospitales certificados

- Atender toda urgencia calificada, aunque no se demuestre derechohabientica o se carezca de recursos económicos, no hacerlo es ilícito

- Asegurar que la atención del enfermo la continuará otro colega

- En situación de huelga, catástrofe, epidemia o grave riesgo para el médico, no abandonar a su enfermo 


\section{BiBLIOGRAFÍA}

1. Comisión Nacional de Bioética [Internet]. ¿Qué es Bioética? México: Secretaría de Salud; 2014 [actualizado: 07-09-2015; acceso:2019-jun-22]. Disponible en: http://www. conbioetica-mexico.salud.gob.mx/interior/queeslabioetica. html

2. Reich WT. Encyclopedia of bioethics. Nueva York: Macmillan; 1978. p. 36.

3. Rodríguez WF, Ortega CJ, Ramírez AJ. Bioética y su aprendizaje en el equipo de salud. Acta Med. 2018; 16 (1): 5.

4. Asociación Médica Mundial. Declaración de Ginebra [Internet]. World Medical Association; 2018 [acceso: 2019mayo-18]. Disponible en: https://www.wma.net/es/policiespost/declaracion-de-ginebra/

5. Fernández CS. El Acto Médico: Errory la mala praxis. Boletín CONAMED-OPS. 2016; 5: 3-6.

6. VeraCO.Aspectoséticosylegales en elacto médico. Rev Med La Paz; 2013.19 (2): $73-82$ [acceso: 2020-ene-11]. Disponible en: http://www.scielo.org.bo/scielo.php?script=sci_ arttext\&pid=S1726-89582013000200010\&lng=es

7. Domínguez MO, Ruíz HF. La Bioética en México: Breve Bosquejo Histórico. [Internet]. México: Bioeticlass; 2020 [acceso: 2020-mar-10]. Disponible en http://www. bioeticlass.com/2020/02/7-la-bioetica-en-mexico-brevebosquejo.html

8. Aguilera PR, Prado MR, López SR. Bioética, bioderecho y biopolítica: problemas actuales. Monterrey, México: Universidad Autónoma de Nuevo León; 2013.

9. Ordóñez AY. Perder un imperio por un simple clavo, cuestiones de ética médica. [Internet]. México: Bioeticlass; 2019 [acceso: 2020-ene-19]. Disponible en. http://wnw.bioeticlass. com/2019/09/6-perder-un-imperio-por-un-simple-clavo.html

10. Hardy PA, Rovero LJ. Moral, ética y bioética. Un punto de vista práctico. Rev Medicina e Investigación. 2015; 3 (1): 7984.

11. Secretaría de Salud. Código de Conducta para el personal de la Secretaría de Salud [Internet]. México: Secretaría de Salud; 2018 [acceso: 2019-jun-22]. Disponible en: https://www.gob.mx/salud/articulos/nuevo-codigode-conducta-para-el-personal-de-la-secretaria-desalud?idiom=es
12. Martínez-Gómez JA. Ética profesional y deontología médica: una reflexión sobre el estado de la cuestión en Cuba. Rev Bioética. 2015; 15 (3): 4-13.

13. Colegio Médico del Perú. Código de Ética y Deontología [Internet]. Código de Ética y Deontología. Colegio Médico del Perú; 2017 [acceso: 2019-jun-22]. Disponible en: http:// repositorio.cmp.org.pe/bitstream/CMP/25/7/CODICO_DE_ ETICA_CMP.pdf

14. Gómez GC, Gómez GM. Ética perinatal. México: Ediciones Gómez Gómez; 2007.

15. Asociación Médica Mundial. Código Internacional de Ética Médica [Internet]. World Medical Association; 2017 [acceso: 2019-mayo-18]. Disponible en: https://www.wma.net/es/ policies-post/codigo-internacional-de-etica-medica/

16. Secretaría de Salud. Código de Bioética para el Personal de Salud [Internet]. México: Secretaría de Salud; 2002 [acceso: 2019-mayo-18]. Disponible en: http://www.salud.gob.mx/ unidades/cdi/documentos/DOCSAL7470.html

17. Secretaría de Salud. Diario Oficial de la Federación: Ley General de Salud. México: 7 febrero 1984. Secretaría de Salud; 2018 [publicado 1984-02-07; actualizado 2018-07-12]. Disponible en: http://www.diputados.gob.mx/LeyesBiblio/ pdf/142_241218.pdf

18. Secretaría de Salud. Normas Oficiales Mexicanas. [Internet] México: Gobierno de México; 2015 [acceso: 2019-junio-25]. Disponible en: https://www.gob.mx/salud/en/documentos/ normas-oficiales-mexicanas-9705

19. Comisión Nacional de Arbitraje Médico. Información sobre la CONAMED para los Profesionales de la Salud [Internet]; 2016 [acceso: 2019-junio-25]. Disponible en http://www. conamed.gob.mx/prof_salud/pdf/funciones.pdf

20. Comisión de Bioética del Consejo Estatal de Estudiantes de Medicina. Guía de Recomendaciones Éticas para el Estudiante de Medicina [Internet]. Comisión de Bioética del Consejo Estatal de Estudiantes de Medicina; 2009 [acceso: 2019-junio-20]. Disponible en: http://aebioetica. org/archivos/guiaestudianmed.pdf

21. Cisneros-Conzález MS, García-Resendiz 1O. Códigos de Bioética para el Profesional de la Salud [Internet]. México: Bioeticlass; 2020 [acceso: 2020-mar-29]. Disponible en http://www.bioeticlass.com/2020/03/2-codigos-debioetica-para.html 